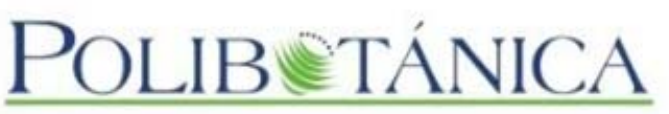

\title{
Polibotánica
}

ISSN electrónico: 2395-9525

polibotanica@gmail.com

Instituto Politécnico Nacional

México

http:www.polibotanica.mx

\section{EXPLORACIÓN Y \\ CARACTERIZACIÓN DE \\ POBLACIONES DE Carica papaya \\ Y Jacaratia mexicana: \\ PARTICULARIDADES DE FRUTOS}

\section{EXPLORATION AND \\ CHARACTERIZATION OF \\ POPULATIONS OF Carica papaya \\ AND Jacaratia mexicana: \\ PARTICULARITIES OF FRUITS}

Álvarez-Hernández, J.C.; J. Z. Castellanos-Ramos, y C.L. Aguirre-Mancilla.

EXPLORACIÓN Y CARACTERIZACIÓN DE POBLACIONES DE Carica papaya Y Jacaratia mexicana: PARTICULARIDADES DE FRUTOS.

EXPLORATION AND CHARACTERIZATION OF POPULATIONS OF Carica papaya AND Jacaratia mexicana: PARTICULARITIES OF FRUITS.

Polibetánica

Instituto Politécnico Nacional
Núm. 48: 43-57 México. Julio 2019

DOI: $10.18387 /$ polibotanica.48.4

(c) (i) Este es un artículo de acceso abierto bajo la licencia Creative Commons 4.0 Atribución-No Comercial (CC BY-NC 4.0 Internacional). 


\section{EXPLORACIÓN Y CARACTERIZACIÓN DE POBLACIONES DE Carica papaya Y Jacaratia mexicana: PARTICULARIDADES DE FRUTOS}

\section{EXPLORATION AND CHARACTERIZATION OF POPULATIONS OF Carica papaya AND Jacaratia mexicana: PARTICULARITIES OF FRUITS}

Álvarez-Hernández, J.C.;

J. Z. Castellanos-Ramos, y

C.L. Aguirre-Mancilla

EXPLORACIÓN Y

CARACTERIZACIÓN DE

POBLACIONES DE Carica

papaya Y Jacaratia mexicana:

PARTICULARIDADES DE FRUTOS

EXPLORATION AND

CHARACTERIZATION OF

POPULATIONS OF Carica

papaya AND Jacaratia mexicana:

PARTICULARITIES OF FRUITS

\section{POLIBETÁNICA}

Instituto Politécnico Nacional

Núm. 48: 43-57. Julio 2019

DOI:

10.18387/polibotanica.48.4
J.C. Álvarez-Hernández / alvarez.juan@ inifap.gob.mx Área agrícola, Programa de Investigación Frutales, Campo Experimental Valle de Apatzingán, CIRPAC-INIFAP, Km 17.5 Carretera Apatzingán-Cuatro Caminos. CP 60781. Antúnez, Parácuaro Michoacán, México. Tel. 018000882222 Ext. 84620.

J. Z. Castellanos-Ramos
C.L. Aguirre-Mancilla
División de Estudios de Posgrado e Investigación,
Instituto Tecnológico de Roque, Tecnológico Nacional de México, Km.8
Carretera Celaya-Juventino Rosas, Apartado Postal 508. CP 38110. Celaya,
Guanajuato, México. Tel. O1 4616115903 Ext. 135.

RESUMEN: México conserva gran diversidad de parientes silvestres de plantas cultivadas; por ejemplo, diferentes especies de solanáceas, cucurbitáceas y caricáceas. En esta última, los géneros Carica y Jacaratia son los más notables por la similitud de los frutos, pero aun pobremente estudiados. Estas poblaciones desarrollan bajo presión edafo-climática y fitosanitaria. Dado el interés de aprovechar estos materiales, el objetivo fue identificar, colectar y caracterizar germoplasma de C. papaya y $J$. mexicana. Se identificaron poblaciones en cuatro estados de México, los sitios fueron caracterizados y en las poblaciones se colectaron frutos. Se registró tamaño, peso y número de semillas, y germinación. Los datos se analizaron por estadísticas descriptivas, análisis de varianza y comparación de medias, la germinación fue porcentual. Además, se realizó un análisis multivariado. Se localizaron 10 poblaciones, en altitudes menores a $900 \mathrm{~m}$ s.n.m., en climas cálido subhúmedo y semiseco. Se distinguieron frutos pequeños que correspondieron a poblaciones de papaya silvestre y frutos grandes, estos últimos superaron en más de $100 \%$ en tamaño con respecto a los frutos pequeños. Los bonetes difieren estructuralmente por las costillas, pero su dimensión es cercana a las poblaciones de papaya semicultivada. La germinación rondó del $28.2 \%$ al $68.8 \%$ y ocho de 10 poblaciones germinó a los 12 días. Se concluye, el germoplasma de las poblaciones silvestres presentó similitud en las medias registradas, a diferencia del germoplasma de las poblaciones semicultivadas, que orienta a prever que se encuentran en proceso de domesticación. La germinación porcentualmente fue baja y varió en el tiempo de observación. El análisis multivariado permitió clasificar tres grupos conjugados y uno independiente.

Palabras clave: Bonete, Caricáceas, papaya, parientes silvestres, poblaciones.

ABSTRACT: Mexico conserves a great diversity of wild relatives of cultivated plants; for example, different Solanaceae, Cucurbitaceae and Caricaceae species families. In the latter, the Carica and Jacaratia genus are the most notable for the similarity of the fruits, but still poorly studied. These populations develop under edapho-climatic and phytosanitary pressure. Due to the interest to take advantage of these materials, the objective was to identify, collect and characterize $C$. papaya and $J$. mexicana germplasm. Populations were identified in four Mexican states, sites were characterized and fruits were collected in the populations. Size, weight and number of 
seeds were registered, and germination. The data was analyzed by descriptive statistics, analysis of variance and comparison of means, germination was percentage. Furthermore, multivariate analysis was performed. Ten populations were located, at altitudes lower than 900 meters above sea level, in subhumid and semi-dry warm climates. Small fruits were distinguished that correspond to populations of wild papaya and large fruits, the latter exceeding by more than $100 \%$ in size with respect to small fruits. The "bonetes" differ structurally by the ribs, but their dimension is close to the populations of semi-cultivated papaya. Germination ranged from $28.2 \%$ to $68.8 \%$ and eight of 10 populations germinated at 12 days. It is concluded that the germplasm of the wild populations showed similarity in the registered means, unlike the germplasm of the semi-cultivated populations, which suggests that they are in the process of domestication. The percentage germination was low and varied in the observation time. The multivariate analysis allowed us to classify three conjugated groups and one independent group. Key words: Bonete, Caricaceas, papaya, populations, wild relatives.

\section{INTRODUCCIÓN}

Con una privilegiada posición geográfica, México al igual que otros países de climas tropicales y subtropicales conserva gran diversidad de parientes silvestres de especies cultivadas. Estos materiales se preservan en hábitat poco perturbados y en campos agrícolas en donde son considerados maleza y reservorios de plagas y enfermedades; sin embargo, las constantes actividades humanas amenazan su permanencia para la estabilidad de los ecosistemas (Delgado et al., 2016). No obstante, los parientes silvestres de cultivos deben considerarse como la principal fuente de germoplasma para el mejoramiento de los cultivos y desarrollo agrícola (Hunter \& Heywood, 2011). Algunos ejemplos representativos son especies hortícolas de las familias Solanaceae, Cucurbitaceae y Caricaceae (Cortez-Madrigal \& García-Ruiz, 2007). Particularmente en la familia Caricaceae, de los seis géneros identificados y documentados (Badillo, 2000; Antunes et al., 2014), Carica y Jacaratia son los géneros más notables desde una apreciación agronómica. Estos se encuentran distribuidos de forma natural en América tropical, desde el norte al sur de México pasando por diversos climas y altitudes, desde nivel del mar y hasta 2700 m s.n.m.; sin embargo, la información sobre caracterización básica es limitada (Acosta, 2002), tanto en las poblaciones silvestres como en las semicultivadas, de sitios aislados no poblados, como en Yucatán, México (Fuentes \& Santamaría, 2014).

Por su porte estructural, las especies Carica papaya L. y Jacaratia mexicana A. DC. son fácilmente distinguibles. Generalmente $C$. papaya no ramifica, solo si se pierde el meristemo apical o si la planta es longeva, esta tiende a la ramificación (Kubitzki, 2003). En cuanto forma, tamaño, color y adaptación de las plantas al medio en que desarrollan, existe amplia variabilidad en poblaciones silvestres (Chávez-Pesqueira et al., 2014; Delgado et al., 2016). Al presentar flores masculinas y femeninas en diferentes individuos, las poblaciones son dioicas (Antunes \& Renner, 2012). La pulpa de los frutos maduros puede variar de color amarillo, naranja o incluso rojo (Aikpokpodion, 2012), en cuanto forma del fruto, redondo, ovalado y elíptico; específicamente, en plantas semicultivadas, los frutos son elongados y cilíndricos con extremidades cónicas, lo cual es importante para explorar fines productivos a través de la hibridación y selección de líneas (Rodriguez et al., 2014).

Por su parte, según la edad J. mexicana presenta arboles entre 5 y $25 \mathrm{~m}$ de altura, con un tronco robusto que alcanza hasta $1 \mathrm{~m}$ de diámetro en la base, angostándose hacia la punta, con ramas delgadas y horizontales (García \& Linares, 2012). Como especie dioica, la floración ocurre durante la estación seca, etapa en que los arboles pierden su follaje (Bullock, 1992). También se ha observado que las plantas clasificadas como masculinas pueden presentar tres tipos de flores, estaminada, pistilada y perfecta (hermafrodita; Aguirre et al., 2009). Los frutos aparecen solitarios, de forma ovoide, esferoide, elipsoide o cónica, la base puede ser redondeada y el pericarpio surcado o acostillado; maduros son verde amarillento, sujetos con pedúnculo alargado (García \& Linares, 2012). 
En las poblaciones silvestres de las especies mencionadas, es posible identificar poblaciones de plantas que desarrollan bajo presión edafo-climática y fitosanitaria, con características sobresalientes, que pudiesen ser factibles de aprovecharse en la mejora de los cultivos (Niklas \& Marler, 2007); sea mediante la hibridación o como porta-injertos de variedades cultivadas. Sin embargo, pocos estudios existen relacionados, algunos de ellos iniciados por la Red Papaya en 2008, iniciativa promovida por el Sistema Nacional de Recursos Fitogenéticos (SINAREFI) cuyos avances fueron notables (SNICS-SAGARPA, 2013). Por ello, con el interés por conocer y conservar de manera sustentable poblaciones silvestres y asilvestradas en zonas del territorio nacional se planteó el objetivo de identificar, colectar y caracterizar el germoplasma silvestre y semi-cultivado de C. papaya y J. mexicana en regiones de cuatro estados de México.

\section{MATERIAL Y MÉTODOS}

En periodos de septiembre de 2016 a abril de 2017 se realizaron recorridos de campo en zonas accesibles y seguras, con el fin de identificar poblaciones o ecotipos de C. papaya y $J$. mexicana. Los sitios fueron seleccionados con conocimiento previo basado en información de agricultores de los Estados de Michoacán, Quintana Roo, Tabasco y Nayarit. Mediante un geoposicionador satelital (GPS), marca Garmin ${ }^{\circledR}$ serie Etrex se obtuvieron registros de latitud, longitud y altura sobre el nivel del mar. Además, variables edafoclimáticas y de vegetación de los sitios fueron complementadas con información documental de los Anuarios Estadísticos y Geográficos (INEGI, 2016a; INEGI, 2016b; INEGI, 2016c; INEGI, 2016d).

Por las características de las plantas, las poblaciones se identificaron como: papaya silvestre, con cinco poblaciones; papaya semicultivada, con tres poblaciones; y bonete (J. mexicana) con dos poblaciones; en total 10 poblaciones. En estas plantas, por su origen y condición diversa, la colecta de frutos en estado de sazón a madurez se ajustó a un número determinado, que dependió de la disponibilidad en la planta, quedando de la siguiente forma, en las cinco poblaciones de papaya silvestre y uno de los materiales de papaya semicultivada identificada como Antúnez 1 se colectaron 15 frutos por cada población. En los dos materiales restantes de papaya semicultivada y las dos poblaciones de bonetes solo se colectaron 5 frutos, debido a la disponibilidad en el momento. La variable registrada in situ, fue el número de frutos por racimo; y en frutos colectados fueron: el diámetro ecuatorial, el diámetro polar, espesor de pulpa, peso de fruto y número de semillas/fruto. Las dos primeras se determinaron mediante un vernier graduado marca Pretul ${ }^{\circledR}$ modelo Vernier 2000; el peso de fruto se obtuvo con báscula digital marca Denver Instrument Company Modelo AA-160. Las semillas extraídas fueron sometidas a tratamiento de lavado y secado para su posterior germinación. Teniendo en cuenta que la tasa de germinación entre plantas silvestres, cultivadas o semicultivadas suele ser diferente (Meletti \& Bruckner, 2001) se estableció una prueba de germinación para caracterizar las semillas de los diferentes materiales compuesto inicialmente por lotes de 500 semillas. La germinación se basó en dos pasos, primero se remojó la semilla en agua por tiempo de siete días, se cambió el agua diariamente. Conociendo el número inicial de semilla y por diferencia se registró la semilla que permaneció en el fondo, y se eliminó la flotante, considerada como semilla vana. El segundo paso consistió en rescatar la semilla remojada y fue colocada en franelas húmedas por 24 días a temperatura ambiente $\left(27 \pm 3^{\circ} \mathrm{C}\right)$, mediante revisiones diarias se registró la semilla germinada y su distribución en el tiempo fue porcentual.

La caracterización de los sitios se apoyó en equipo y literatura especializada (INEGI, 2016a; INEGI, 2016b; INEGI, 2016c; INEGI, 2016d). La caracterización de frutos fue analizada mediante estadísticas descriptiva (media y desviación estándar), análisis de varianza y la separación de medias con Tukey $(P=0.05)$. Además, se realizó un análisis multivariado de componentes principales (CP) y conglomerado con base en la matriz 7x10, correspondiente a características de frutos y poblaciones, respectivamente, y se correlacionó para estimar el grado de asociación. El paquete estadístico utilizado fue SAS versión 9.3 (2002). En cuanto a la prueba de germinación el resultado se interpretó en base al porcentaje de germinación. 


\section{RESULTADOS}

En los estados explorados se localizaron 10 sitios con germoplasma de interés (cuadro 1). Debido a la facilidad y cercanía para trasladarse de un sitio a otro, Michoacán fue el estado mayormente explorado. También, a pesar de que solo se registraron tres sitios representativos de los estados de Quintana Roo, Tabasco y Nayarit, es probable que existan más sitios propicios para explorar, ya que estos estados presentaron condiciones para el desarrollo de las poblaciones silvestres. Respecto a características de las poblaciones identificadas se distinguieron tres tipos: papaya silvestre y semicultivada, y los bonetes (fig. 1), en general se ubicaron por debajo de los 900 m s.n.m. (cuadro 1).

La mayoría de las poblaciones se concentraron en climas cálido subhúmedo con precipitación media de $1000 \mathrm{~mm}$ anuales; cálido semiseco con precipitación máxima de $800 \mathrm{~mm}$ anuales, caso de Michoacán, particularmente en la zona conocida como Valle de Apatzingán; y cálido húmedo con precipitación de $2000 \mathrm{~mm}$ anuales, como fue el caso del estado de Tabasco (cuadro 2). El tipo de suelo, parece no ser un factor determinante para el desarrollo de las poblaciones; sin embargo, en la mayoría predominaron los suelos tipo vertisol. La vegetación colindante estuvo influenciada por el aspecto climático, y comprende los tipos de selva mediana sub-caducifolia y la selva baja caducifolia en el clima cálido semiseco (cuadro 2).

En la característica frutos por racimo evaluado in situ, los resultados se muestran en la figura 2 , las poblaciones de papaya silvestre (Aquila, Coahuayana, Q. R., Tabasco y Nayarit), además Antúnez 1, presentan los frutos conformados en racimos, y entre ellos, la población de papaya silvestre Tabasco concentra mayor cantidad de frutos por racimo alcanzando en promedio seis frutos por racimo. En cambio, las poblaciones Mujica y Antúnez 2, correspondientes a los materiales semicultivados, así como los bonetes (poblaciones de Arteaga y Buenos Aires) no presentaron racimos, cuyos frutos aparecen solitarios (fig. 2).

Cuadro 1. Lugares explorados con presencia de poblaciones de C. papaya y J. mexicana.

\begin{tabular}{|c|c|c|c|c|c|}
\hline Estado & Localización (identificación) & Germoplasma & $\begin{array}{c}\text { Altitud } \\
\text { (m s.n.m.) }\end{array}$ & Latitud Norte & Longitud Oeste \\
\hline \multirow[t]{7}{*}{ Michoacán } & Aquila (Aquila) & PS & 202 & $18^{\circ} 35^{\prime} 45^{\prime \prime}$ & $103^{\circ} 29^{\prime} 26^{\prime \prime}$ \\
\hline & Coahuayana (Coahuayana) & PS & 23 & $18^{\circ} 44^{\prime} 30^{\prime \prime}$ & $103^{\circ} 38^{\prime} 27^{\prime \prime}$ \\
\hline & Antúnez, Parácuaro (Antúnez 1) & PSC (porte alto) & 360 & $19^{\circ} 00^{\prime} 46^{\prime \prime}$ & $102^{\circ} 13^{\prime} 50^{\prime \prime}$ \\
\hline & Antúnez, Parácuaro (Antúnez 2) & PSC (hojas "huacas") & 371 & $19^{\circ} 01^{\prime} 11^{\prime \prime}$ & $102^{\circ} 12^{\prime} 01^{\prime \prime}$ \\
\hline & Nueva Italia (Mujica) & PSC (peciolos morados) & 418 & $18^{\circ} 59^{\prime} 30^{\prime \prime}$ & $102^{\circ} 06^{\prime} 22^{\prime \prime}$ \\
\hline & Arteaga (Arteaga) & Bo & 864 & $18^{\circ} 22^{\prime} 26^{\prime \prime}$ & $102^{\circ} 17^{\prime} 42^{\prime \prime}$ \\
\hline & $\begin{array}{l}\text { Buenos Aires, Parácuaro } \\
\text { (Buenos Aires) }\end{array}$ & Bo & 486 & $19^{\circ} 05^{\prime} 01^{\prime \prime}$ & $102^{\circ} 11^{\prime} 19^{\prime \prime}$ \\
\hline Nayarit & Mesillas (Nayarit) & PS & 462 & $21^{\circ} 12^{\prime} 53^{\prime \prime}$ & $105^{\circ} 02^{\prime} 04^{\prime \prime}$ \\
\hline Tabasco & Poblado C 27, Cárdenas (Tabasco) & PS & 11 & $17^{\circ} 59^{\prime} 51^{\prime \prime}$ & $93^{\circ} 34^{\prime} 35^{\prime \prime}$ \\
\hline Quintana Roo & Othón Pompeyo Blanco (Q. R.) & PS & 10 & $18^{\circ} 29^{\prime} 53^{\prime \prime}$ & $88^{\circ} 20^{\prime} 02^{\prime \prime}$ \\
\hline
\end{tabular}

PS = Papaya silvestre; PSC = Papaya semicultivada; Bo $=$ Bonete. 


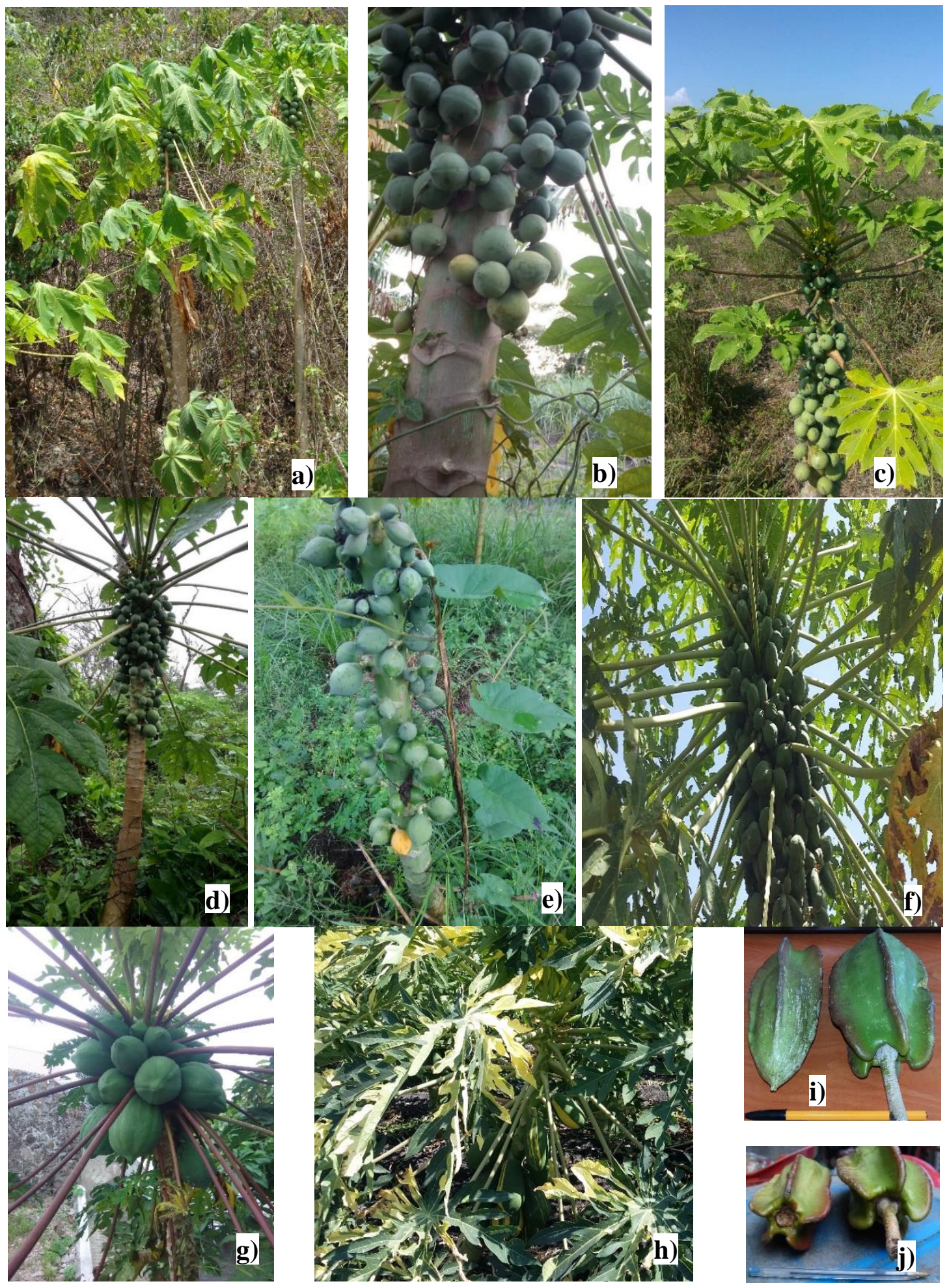

Fig. 1. Germoplasma característico de C. papaya y J. mexicana: poblaciones de papaya silvestre a) Aquila, b) Coahuayana, c) Quintana Roo (Q. R.), d) Tabasco, e) Nayarit; poblaciones de papaya semicultivada f) Antúnez 1, g) Mujica, h) Antúnez 2; poblaciones de bonetes i) Arteaga, j) Buenos Aires. 
Cuadro 2. Variables climáticas, predominancia de suelos y vegetación de poblaciones de C. papaya y J. mexicana.

\begin{tabular}{|c|c|c|c|c|c|}
\hline Población & Clasificación climática & $\begin{array}{c}\text { Temperatura } \\
\text { media anual }\left({ }^{\circ} \mathbf{C}\right)\end{array}$ & $\begin{array}{l}\text { Precipitación } \\
(\mathbf{m m})\end{array}$ & $\begin{array}{l}\text { Tipo de } \\
\text { suelo }\end{array}$ & Vegetación \\
\hline Aquila & $\begin{array}{l}\text { Cálido subhúmedo con } \\
\text { lluvias en verano }\end{array}$ & $24-26$ & $1000-1200$ & $\begin{array}{l}\text { Luvisol y } \\
\text { leptosol }\end{array}$ & $\begin{array}{l}\text { Selva mediana } \\
\text { subcaducifolia }\end{array}$ \\
\hline Coahuayana & $\begin{array}{l}\text { Cálido subhúmedo con } \\
\text { lluvias en verano }\end{array}$ & $26-28$ & $1000-1200$ & Phaeozem & $\begin{array}{l}\text { Selva mediana } \\
\text { subcaducifolia }\end{array}$ \\
\hline Antúnez 1 & Cálido semiseco & $26-28$ & $700-800$ & Vertisol & $\begin{array}{l}\text { Selva baja } \\
\text { caducifolia }\end{array}$ \\
\hline Antúnez 2 & Cálido semiseco & $26-28$ & $700-800$ & Vertisol & $\begin{array}{l}\text { Selva baja } \\
\text { caducifolia }\end{array}$ \\
\hline Mujica & Cálido semiseco & $26-28$ & $700-800$ & Vertisol & $\begin{array}{l}\text { Selva baja } \\
\text { caducifolia }\end{array}$ \\
\hline Arteaga & $\begin{array}{l}\text { Cálido subhúmedo con } \\
\text { lluvias en verano }\end{array}$ & $22-24$ & $800-1000$ & $\begin{array}{l}\text { Leptosol y } \\
\text { luvisol }\end{array}$ & $\begin{array}{l}\text { Selva mediana } \\
\text { subcaducifolia }\end{array}$ \\
\hline Buenos Aires & Cálido semiseco & $26-28$ & $700-800$ & Vertisol & $\begin{array}{l}\text { Selva baja } \\
\text { caducifolia }\end{array}$ \\
\hline Nayarit & $\begin{array}{l}\text { Cálido subhúmedo con } \\
\text { lluvias en verano }\end{array}$ & $22-24$ & $1000-1200$ & Leptosol & $\begin{array}{l}\text { Selva mediana } \\
\text { subcaducifolia }\end{array}$ \\
\hline Tabasco & $\begin{array}{l}\text { Cálido húmedo con lluvias } \\
\text { en verano }\end{array}$ & $26-28$ & $1500-2000$ & Vertisol & $\begin{array}{l}\text { Selva mediana } \\
\text { subcaducifolia }\end{array}$ \\
\hline Q. R. & $\begin{array}{l}\text { Cálido subhúmedo con } \\
\text { lluvias en verano }\end{array}$ & $26-28$ & $1200-1500$ & Vertisol & $\begin{array}{l}\text { Selva mediana } \\
\text { subcaducifolia }\end{array}$ \\
\hline
\end{tabular}

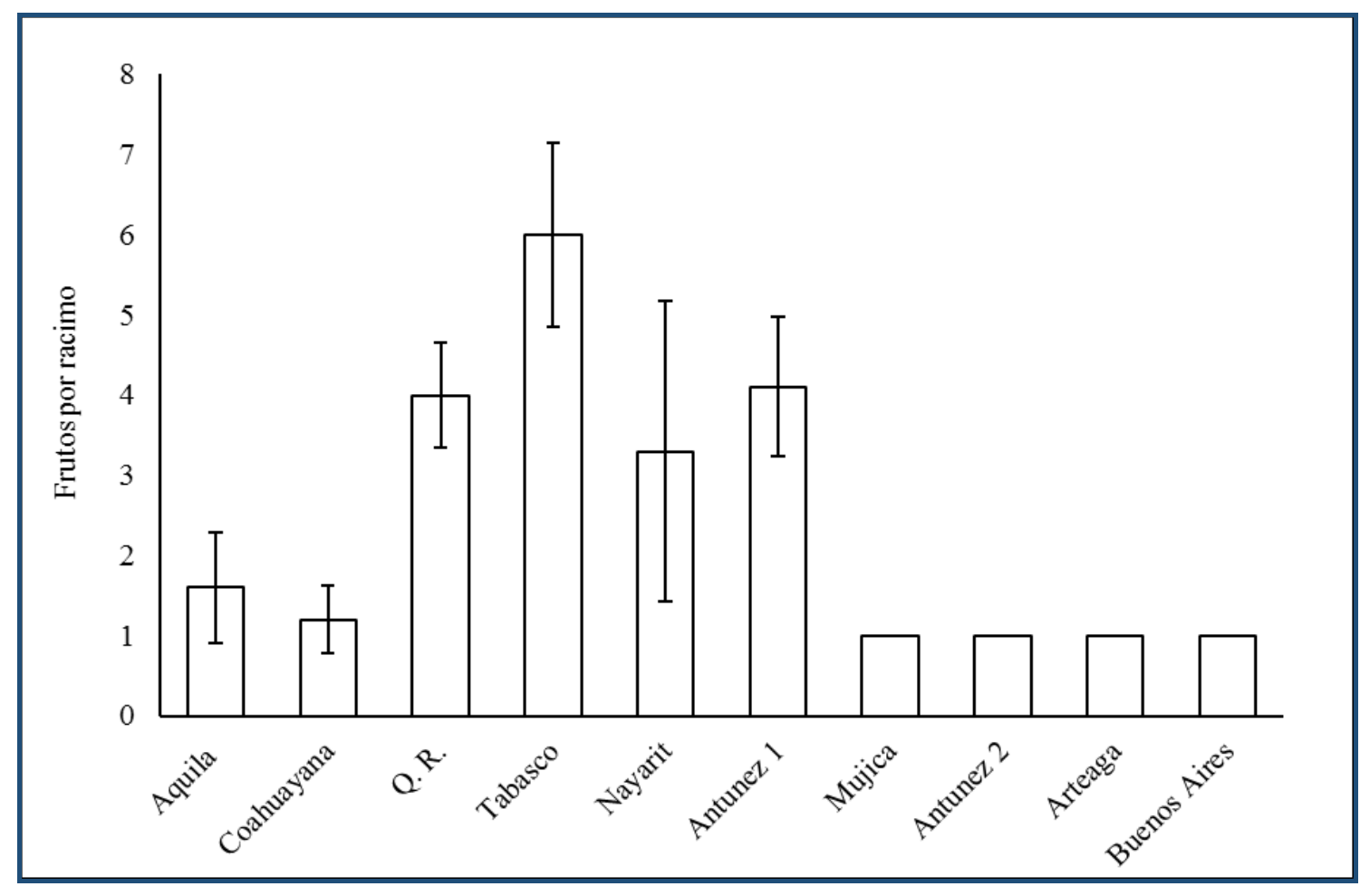

Fig. 2. Conformación de frutos por planta de las poblaciones de estudio. 
La caracterización de los frutos se presenta en el cuadro 3, según el análisis estadístico, las poblaciones de C. papaya por su tamaño de fruto se distinguieron claramente dos tipos, frutos pequeños y frutos grandes. Las poblaciones de frutos pequeños corresponden a las poblaciones de papaya silvestre (Aquila, Coahuayana, Q. R., Tabasco y Nayarit), el tamaño de fruto varió de $6.78 \mathrm{~cm}$ a $7.28 \mathrm{~cm}$ de diámetro polar y $6.62 \mathrm{~cm}$ a $7.19 \mathrm{~cm}$ de diámetro ecuatorial, en comparación con las poblaciones de fruto grande, las cuales superan en más de $100 \%$ el tamaño (cuadro 3). Esta tendencia, también se vio reflejada en la variable ancho de pulpa, ya que proporcionalmente las poblaciones Mujica, Antúnez 2 e incluso Antúnez 1, aun cuando esta última fue más semejante a los materiales silvestres en otras variables registradas. Las poblaciones de J. mexicana (Arteaga y Buenos Aires) a pesar de que el fruto difiere estructuralmente, presenta valores ligeramente superiores a $20 \mathrm{~cm}$ de diámetro polar y entre 8 $\mathrm{cm}$ y $11 \mathrm{~cm}$ de diámetro ecuatorial, pero en la variable ancho de pulpa, su dimensión es cercana a las poblaciones de papaya semicultivada (cuadro 3 ).

Cuadro 3. Tamaño, peso y número de semillas de frutos colectados en poblaciones de C. papaya y J. mexicana.

\begin{tabular}{lccccc}
\hline \multirow{2}{*}{ Población } & \multicolumn{5}{c}{ Variables } \\
\cline { 2 - 6 } & $\begin{array}{c}\text { Diámetro polar } \\
(\mathbf{c m})\end{array}$ & $\begin{array}{c}\text { Diámetro } \\
\text { ecuatorial }(\mathbf{c m})\end{array}$ & $\begin{array}{c}\text { Ancho de } \\
\text { pulpa }(\mathbf{c m})\end{array}$ & $\begin{array}{c}\text { Número de } \\
\text { semillas/fruto }\end{array}$ & Peso de fruto (g) \\
\hline Aquila (P) & $6.82 \pm 0.75 \mathrm{e}^{\dagger}$ & $6.64 \pm 0.70 \mathrm{e}$ & $0.66 \pm 0.11 \mathrm{f}$ & $269.20 \pm 50.32 \mathrm{ab}$ & $72.67 \pm 8.89 \mathrm{ef}$ \\
Coahuayana (P) & $6.78 \pm 0.51 \mathrm{e}$ & $6.62 \pm 0.44 \mathrm{e}$ & $0.86 \pm 0.51 \mathrm{ef}$ & $275.67 \pm 43.14 \mathrm{a}$ & $58.53 \pm 7.42 \mathrm{f}$ \\
Antúnez 1 (P) & $11.86 \pm 0.69 \mathrm{~d}$ & $10.65 \pm 0.71 \mathrm{c}$ & $1.09 \pm 0.12 \mathrm{de}$ & $316.07 \pm 38.47 \mathrm{a}$ & $140.87 \pm 10.61 \mathrm{c}$ \\
Antúnez 2 (P) & $41.00 \pm 2.34 \mathrm{a}$ & $24.00 \pm 2.54 \mathrm{a}$ & $3.66 \pm 0.20 \mathrm{a}$ & $55.60 \pm 24.85 \mathrm{~d}$ & $829.60 \pm 77.33 \mathrm{a}$ \\
Mujica (P) & $25.60 \pm 3.80 \mathrm{~b}$ & $15.46 \pm 1.55 \mathrm{~b}$ & $3.04 \pm 0.20 \mathrm{~b}$ & $110.00 \pm 30.48 \mathrm{~d}$ & $567.60 \pm 71.12 \mathrm{~b}$ \\
Arteaga (B) & $20.28 \pm 3.24 \mathrm{c}$ & $11.47 \pm 2.68 \mathrm{c}$ & $1.44 \pm 0.20 \mathrm{~cd}$ & $208.40 \pm 33.38 \mathrm{c}$ & $112.80 \pm 15.25 \mathrm{~cd}$ \\
Buenos Aires (B) & $20.74 \pm 0.78 \mathrm{c}$ & $8.78 \pm 0.94 \mathrm{~d}$ & $1.48 \pm 0.08 \mathrm{c}$ & $211.80 \pm 37.94 \mathrm{bc}$ & $109.40 \pm 8.76 \mathrm{cde}$ \\
Nayarit (P) & $7.04 \pm 0.39 \mathrm{e}$ & $6.90 \pm 0.40 \mathrm{e}$ & $0.70 \pm 0.07 \mathrm{f}$ & $295.07 \pm 31.70 \mathrm{a}$ & $75.47 \pm 15.36 \mathrm{ef}$ \\
Tabasco (P) & $7.28 \pm 0.26 \mathrm{e}$ & $7.19 \pm 0.30 \mathrm{ed}$ & $0.69 \pm 0.07 \mathrm{f}$ & $321.80 \pm 16.92 \mathrm{a}$ & $87.60 \pm 5.81 \mathrm{def}$ \\
Q. R. (P) & $6.82 \pm 0.42 \mathrm{e}$ & $6.67 \pm 0.40 \mathrm{e}$ & $0.75 \pm 0.09 \mathrm{ef}$ & $274.60 \pm 35.21 \mathrm{a}$ & $67.13 \pm 8.83 \mathrm{f}$ \\
DMS & 1.93 & 1.51 & 0.35 & 58.05 & 36.83 \\
C.V. & 10.83 & 10.82 & 20.44 & 13.78 & 16.34 \\
Significancia & $* * *$ & $* * *$ & $* * *$ & $* *$ & $* * *$ \\
\hline
\end{tabular}

$\mathrm{P}=$ Papaya, $\mathrm{B}=$ Bonete; ${ }^{\dagger}$ Medias \pm desviación estándar dentro de columna seguida de la misma letra no difiere estadísticamente (Tukey, 0.05); D.M.S. $=$ Diferencia mínima significativa; C.V. $=$ Coeficiente de variación; $* * * P \leq 0.0001$.

El número de semillas por fruto se registró en menor cantidad para las poblaciones de Mujica y Antúnez 2 con 110 y 55.6 semillas, respectivamente; sin embargo, el conjunto de las poblaciones de papaya silvestre, así como Antúnez 1, Arteaga y Buenos Aires, estadísticamente mostraron mayor cantidad de semilla, oscilando entre 200 y 300 semillas por fruto; para el caso de papaya semicultivada, su estado refleja un comportamiento de líneas en proceso de domesticación. Por último, en la variable peso de fruto, su comportamiento está muy relacionado con el tamaño de fruto, ancho de pulpa y número de semillas, por lo que se distinguieron los frutos chicos y los frutos grandes (cuadro 3 ).

Con excepción de la población Antúnez 2, todas las poblaciones presentaron porcentajes bajos de semilla vana, que fluctuaron de 0 y $6.6 \%$, incluidos las poblaciones de bonetes, ya que no manifestaron esa condición. Asimismo, en su conjunto todas las poblaciones tuvieron germinación baja respecto a las variedades cultivadas que suele ser del $85 \%$ (SNICSSAGARPA, 2014). Los materiales de papaya silvestre fluctuaron de 33.3 a $57.3 \%$; las papayas semicultivadas de 28.2 a $68.8 \%$ y los bonetes (Arteaga y Buenos Aires) en 41.2 y $38.3 \%$, respectivamente (fig. 3). 
Respecto al comportamiento de la germinación, se distribuyó en el periodo de respuesta que inicio entre 6 y 12 días, y se extendió hasta los 21 y 24 días. Sin embargo, la mayor germinación se registró a los 12 días en la mayoría de las poblaciones, excepto en la población Antúnez 2. Posteriormente la germinación fue disminuyendo conforme avanzó el tiempo (fig. 4).

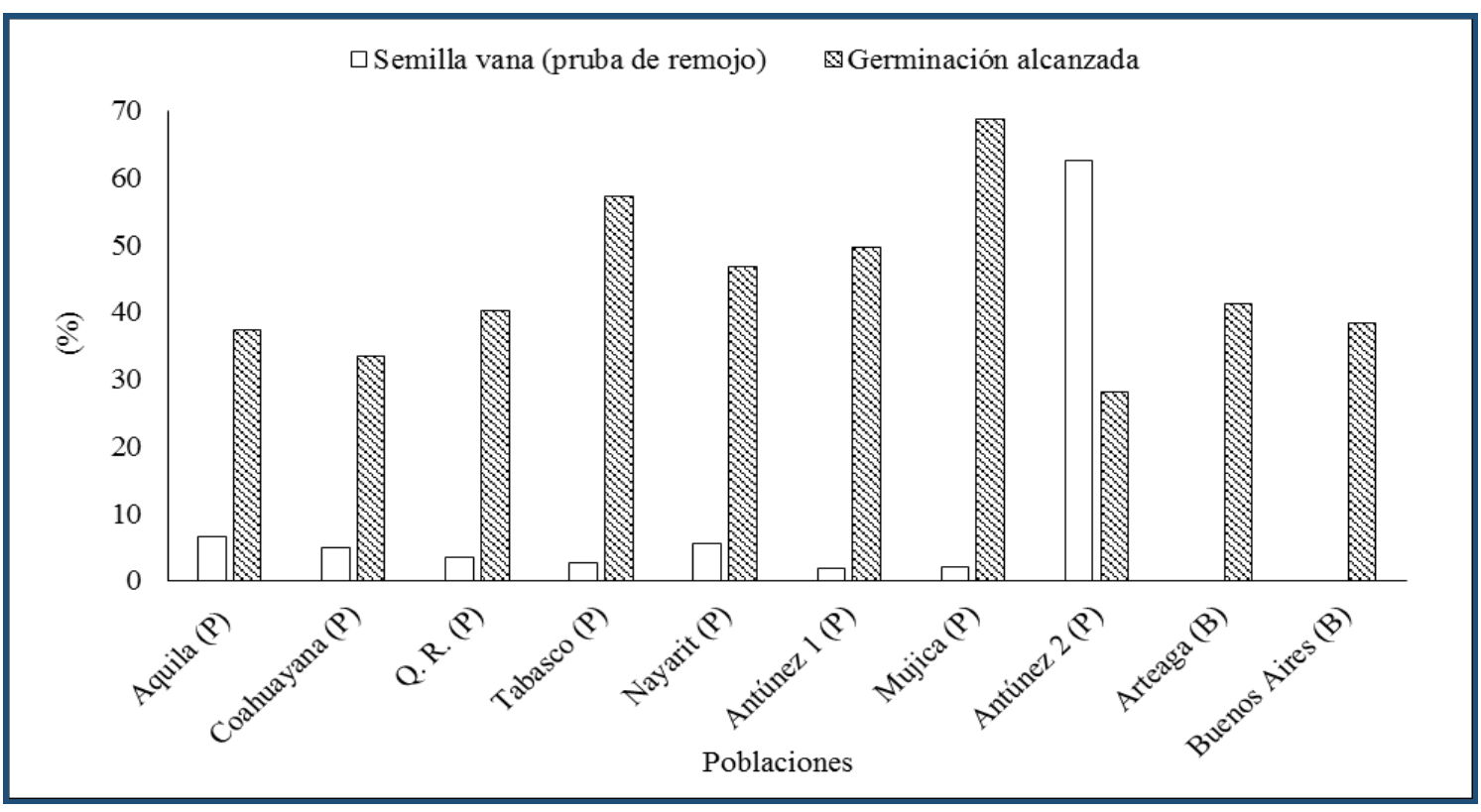

Fig. 3. Semilla vana y germinación alcanzada en poblaciones de C. papaya y J. mexicana.

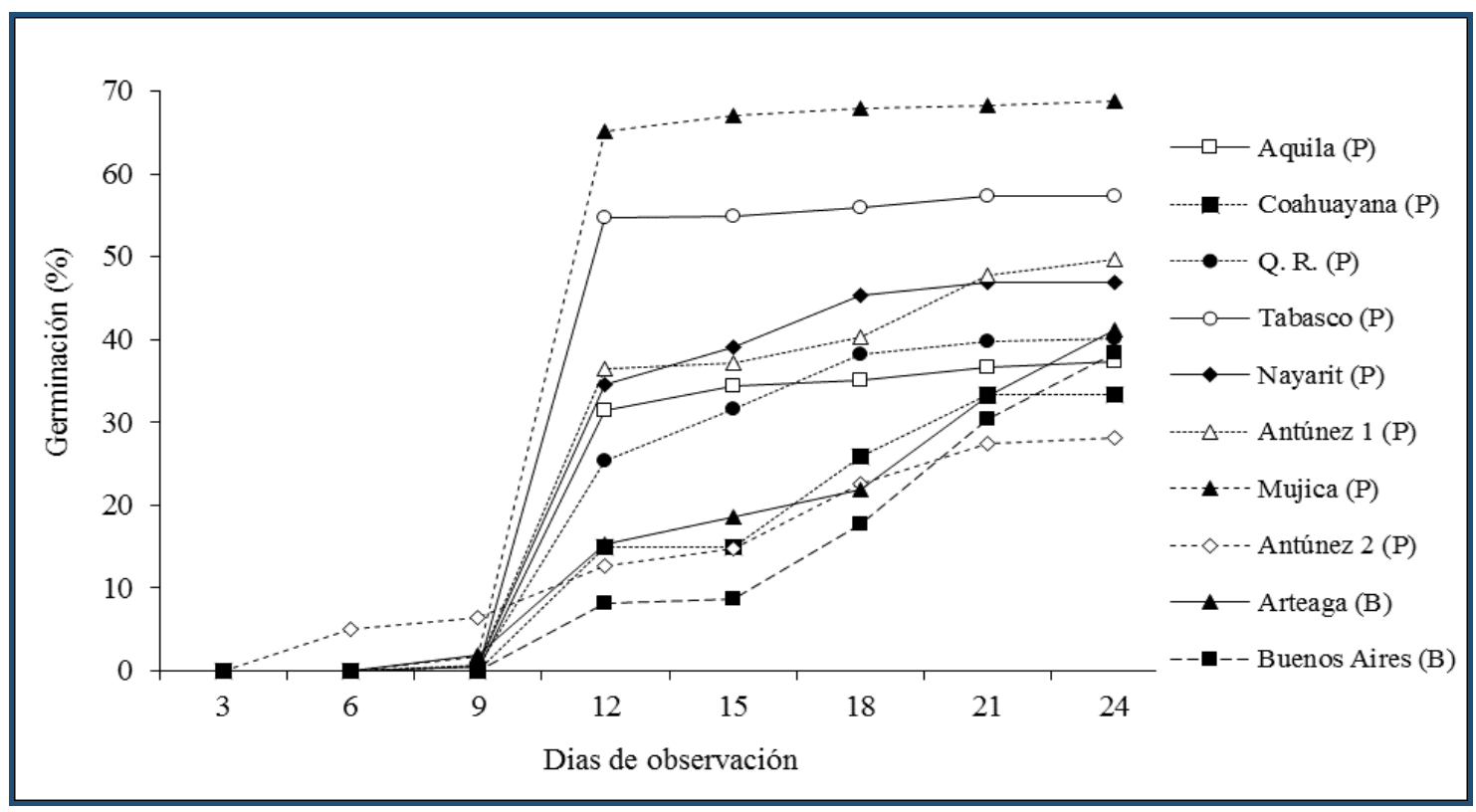

Fig. 4. Variación de la germinación en el tiempo en poblaciones de C. papaya y J. mexicana. 
Por otra parte, el análisis multivariado de componentes principales basado en las siete variables referentes a características de frutos, fue interpretado a partir de los valores propios donde se muestra la varianza individual y acumulada en cada componente del análisis. Asimismo, el valor propio y la varianza de la matriz de correlación. Los valores mostrados indican la variabilidad que está asociada a cada componente principal, y se reduce en medida que se incrementa el número de componente, mostrando en forma acumulada que el primer componente explica cerca del $98.19 \%$ de la variabilidad, el resto concentró el 1.81\% (cuadro 5).

Cuadro 5. Valores propios de las características de frutos y proporción de la variabilidad en 10 poblaciones de C. papaya y J. mexicana.

\begin{tabular}{cccccc}
\hline $\begin{array}{c}\text { Componente } \\
\text { principal }\end{array}$ & \multicolumn{3}{c}{ Matriz varianza-covarianza } & \multicolumn{2}{c}{ Matriz correlación } \\
\cline { 2 - 6 } & $\begin{array}{c}\text { Valor } \\
\text { propio }\end{array}$ & $\begin{array}{c}\text { Varianza } \\
\text { explicada (\%) }\end{array}$ & $\begin{array}{c}\text { Varianza } \\
\text { acumulada (\%) }\end{array}$ & $\begin{array}{c}\text { Valor } \\
\text { propio }\end{array}$ & Varianza (\%) \\
\hline 1 & 78144.4661 & 98.19 & 98.19 & 5.07371 & 72.482 \\
2 & 1293.8391 & 1.63 & 99.81 & 1.23849 & 17.699 \\
3 & 134.7627 & 0.017 & 99.98 & 0.557739 & 7.9677 \\
4 & 14.2607 & 0.002 & 100 & 0.0844144 & 1.2059 \\
\hline
\end{tabular}

Al considerar los dos primeros componentes principales se puede explicar la variabilidad acumulada en las poblaciones. Dado que el primer componente supera el $98 \%$ de toda la varianza (fig. 5), está correlacionado positivamente con el peso de fruto (cuadro 6). El segundo componente representa el $0.0163 \%$ de toda la varianza (fig. 5), esta correlacionado positivamente con el número de semillas (cuadro 6).

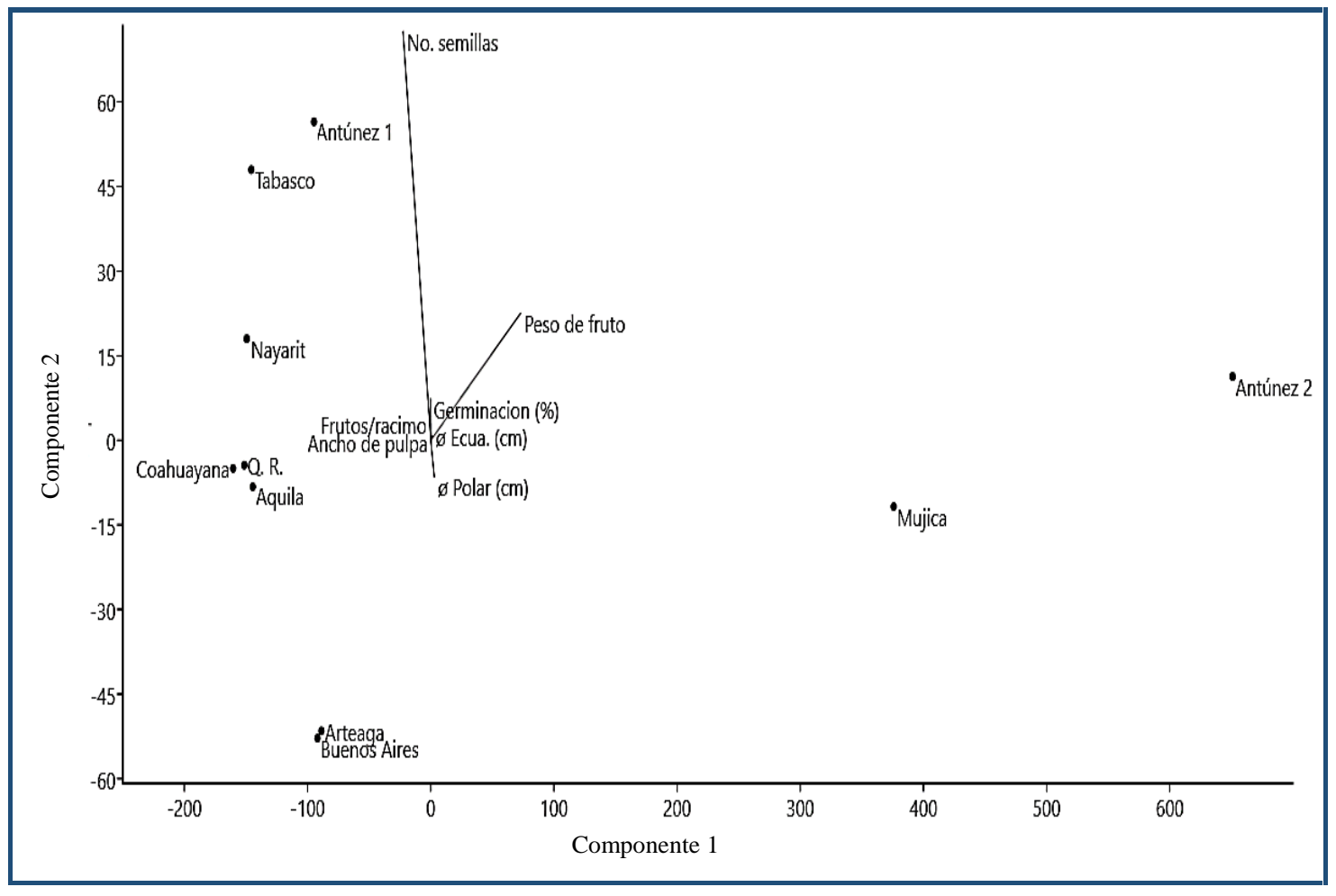

Fig. 5. Dispersión diagramática de poblaciones de Caricáceas y variables de frutos en los componentes principales 1 y 2. 
Cuadro 6. Participación de los componentes principales uno y dos.

\begin{tabular}{lcc}
\hline Variable & Componente principal 1 & Componente principal 2 \\
\hline$\varnothing$ Polar $(\mathrm{cm})$ & 0.037271 & -0.083831 \\
$\varnothing$ Ecua. $(\mathrm{cm})$ & 0.019517 & 0.010573 \\
Ancho de pulpa & 0.0037286 & -0.0046043 \\
Núm. semillas & -0.29377 & 0.94642 \\
Peso de fruto & 0.95494 & 0.2943 \\
Frutos/racimo & -0.0027562 & 0.03755 \\
Germinación $(\%)$ & 0.00024447 & 0.095378 \\
\hline
\end{tabular}

Por su parte, las estructuras de los dos componentes principales se usaron para realizar el análisis de conglomerados. Este análisis se realizó al considerar la estructura de los dos primeros componentes principales ya que explicaron el $99 \%$ de la varianza y pudo facilitar el proceso de identificación de grupos de variantes. Los resultados permitieron evidenciar que a una distancia euclideana de 75 se formaron tres grupos definidos: Arteaga y Buenos Aires; Nayarit, Q. R., Aquila, Coahuayana y Tabasco; y Mujica y Antúnez 2. Antúnez 1 no se formó en ningún grupo (fig. 6).

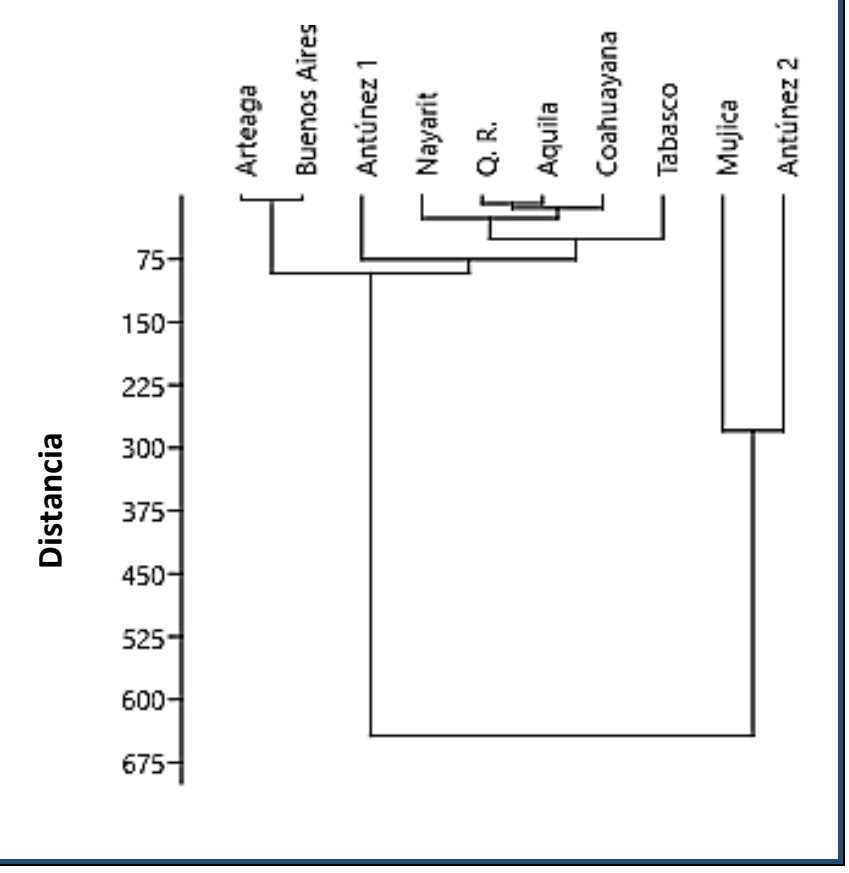

Fig. 6. Dendrograma de siete variables de frutos de Caricaceae, con tres grupos definidos y uno sin definir a distancia euclideana de 75 .

\section{DISCUSIÓN}

A pesar de los esfuerzos por integrar un acervo de información de recursos fitogenéticos de las Caricáceas en México, particularmente en las especies $C$. papaya y J. mexicana, aún está limitado, ya que los reportes formales integran poca información exploratoria y la caracterización de poblaciones, por lo que se desconoce la localización de sitios potenciales 
donde se desarrollan estas especies, aun cuando en muchas partes de México estas especies desarrollan de manera espontánea. A pesar de ello, algunos autores sugieren que esta familia se distribuye en todo el país, derivado de su gran capacidad de adaptación a los diferentes climas (Arias et al., 2010), y estados como Yucatán (Teran \& Rasmussen, 1995), selva de los Tuxtlas, (Paz \& Vázquez-Yanes, 1998), localidades de Baja California Sur (Soriano-Melgar et al., 2016), zonas del estado Guerrero (Delgado et al., 2016), además, otros sitios reportados por el SINAREFI (SNICS-SAGARPA, 2013), Tamaulipas, San Luis Potosí y Campeche, todos con presencia de poblaciones silvestres, que sumados a los cuatro estados reportados en el presente estudio contribuyen a ampliar el conocimiento de la diversidad de esta familia, y en particular para C. papaya. En cambio la localización de poblaciones de J. mexicana en el país no han sido consistentemente documentadas; sin embargo, Bullock (1992) menciona que se distribuye en México, con elevaciones de hasta 1500 m s.n.m. y con presencia en los estados de Veracruz, Nayarit y Michoacán.

Los sitios reportados se localizaron en climas cálidos húmedos y subhúmedos con lluvias en verano, con vegetación de selva baja caducifolia y sub-caducifolia, lo que coincide con lo reportado por Teran \& Rasmussen (1995), que hacen referencia a las poblaciones de estos recursos vegetales en bosques tropicales húmedos y subhúmedos, con plantas silvestres de $C$. papaya en huertos familiares de grupos étnicos de Yucatán, mientras que J. mexicana se desarrolla en ambientes poco modificados, como las poblaciones de Michoacán. Delgado et al. (2016) relacionan las colectas de papaya con climas cálidos, precipitación de $1100 \mathrm{~mm}$, y suelo tipo vertisol pélico, muy semejante con los sitios reportados.

Las poblaciones silvestres de $C$. papaya y $J$. mexicana han sido caracterizadas como poblaciones dioicas, y en casos esporádicos como poblaciones trioicas (Aguirre et al., 2009), según el grado de domesticación y condiciones en las que desarrollan, los frutos suelen aparecer en racimos o individuales, este último más específicos para J. mexicana. También, es de destacar que existe una amplia variabilidad morfológica de frutos. En este estudio con base al tamaño de fruto se distinguieron dos tipos de $C$. papaya y los bonetes, que presentan costillas. Respecto a los frutos pequeños y frutos grandes de papaya, estos últimos principalmente en poblaciones semicultivadas, pero que aún conservan aspectos de las plantas silvestres. Esta variación en la dimensión de frutos la mencionan Soriano-Melgar et al. (2016), que señalan presencia de frutos grandes, aun siendo colectados en una misma localidad. Asimismo, Paz \& Vázquez-Yanes (1998) mencionan que las poblaciones silvestres de C. papaya produce frutos esféricos de $5 \mathrm{~cm}$ a $8 \mathrm{~cm}$ de diámetro, valores similares a los encontrados en los frutos de las poblaciones silvestres, siendo estos de $7.28 \mathrm{~cm}$ a $7.19 \mathrm{~cm}$ diámetro y peso entre $67.13 \mathrm{~g}$ a 87.60 g. Por su parte, Manshardt (2014), reporta poblaciones silvestres con peso de fruto inferior a $100 \mathrm{~g}$ y con pulpa suave, lo cual asemeja a lo encontrado en el presente estudio.

La semilla de los frutos de las plantas de papaya silvestre es una característica distinguible, ya que las plantas silvestres contienen mayor número de semillas que las poblaciones semicultivadas (cuadro 3), pero suele ser de menor tamaño (Paz \& Vázquez-Yanes, 1998), incluso que la semilla de los bonetes. Por lo general, un fruto de C. papaya silvestre contiene hasta 300 semillas (Paz \& Vázquez-Yanes, 1998), en cambio un fruto J. mexicana puede contener hasta 676 semillas por fruto (Aguirre et al., 2009), en las dos poblaciones de bonetes, el número de semillas fue casi una tercera parte (cuadro 3) de lo mencionado. En relación a la germinación, en términos generales la prueba arrojó porcentualmente baja germinación, ya que apenas superó el $50 \%$, aun cuando se dio el tratamiento previo de remojo de semilla, que normalmente se aplica en el manejo de la semilla de papaya cultivada, de igual forma el periodo de respuesta fue prolongado y variable (fig. 4). Al respecto Soriano-Melgar et al. (2016) reportaron porcentajes de germinación entre 10 a 47\%. En cambio, para J. mexicana, Aguirre et al. (2009) reportan baja germinación además esta se da entre 18 y 33 días después de la siembra. 
Por otra parte, el análisis de componentes principales, se requirió al menos de dos componentes para explicar el $99 \%$ de toda la varianza, donde solo el primer componente aportó el $98 \%$ de total de varianza. Con esta información fue posible identificar tres grupos bien definidos y uno sin definir a través del análisis de conglomerados. Como se observó en la figura 6, el grupo uno se integró por las poblaciones de bonetes (Buenos Aires y Arteaga), el grupo dos, por las poblaciones de papaya silvestres (Nayarit, Q. R. Coahuayana, Aquila y Tabasco), el grupo tres, por las poblaciones semicultivadas Mujica y Antúnez 2, por su parte, Antúnez 1, no formó grupo, posiblemente por las características diferentes al resto de las poblaciones; sin embargo, esta variación puede ser aprovechada en otros aspectos productivos a través de la hibridación.

Los estudios exploratorios son importantes, ya que contribuyen sustancialmente en la valoración de la riqueza, además, del privilegio de poseerlos localmente como país. Sin embargo, además de la implementación de un plan de conservación, también es importante una estrategia de multiplicación, distribución y uso de estos recursos, orientados a fortalecer a su homólogo cultivado, aprovechando las características sobresalientes que aun preservan estos parientes silvestres.

\section{Conclusiones}

Se exploraron cuatro estados de México, conteniendo ocho sitios con poblaciones de papaya y dos sitios con poblaciones de bonetes, las cuales desarrollan en ambientes variados. El germoplasma colectado de las poblaciones silvestres denominadas Aquila, Coahuayana, Q. R., Tabasco y Nayarit, presentaron similitud en las medias registradas, incluso a la población Antúnez 1; sin embargo, por su comportamiento puede ser considerado una línea avanzada. En cambio, el comportamiento mostrado del germoplasma de las poblaciones de Mujica y Antúnez 2, orientan a prever que estas poblaciones se encuentran en proceso de domesticación. La germinación de la semilla fue baja y heterogénea y ocurrió en mayor proporción a los 12 días. El análisis multivariado permitió clasificar tres grupos y uno no definido, de estos tanto el grupo de las poblaciones silvestres de papaya como el grupo de los bonetes" fueron los más notables. Los bonetes son materiales interesantes por sus características robustas, y que aún tienen mucho que explorar, por su alto grado de resistencia a factores bióticos y abióticos.

\section{AGRADECIMIENTOS}

Al Tecnológico Nacional de México por el apoyo otorgado para el desarrollo de esta investigación (proyecto: 5841.16-P); y al Instituto Nacional de Investigaciones Forestales, Agrícolas y Pecuarias, por las facilidades concedidas para llevar a cabo esta investigación.

\section{LITERATURA CITADA}

Acosta, C. S. (2002). Plantas vasculares raras, amenazadas, o en peligro de extinción del estado de Oaxaca, un panorama preliminar. Polibotanica, 13, 47-82.

Aguirre, A. Vallejo-Marín, M. Piedra-Malagón, E. M. Cruz-Ortega, R. \& Dirzo, R. (2009). Morphological variation in the flowers of Jacaratia mexicana A. DC. (Caricaceae), a subdioecious tree. Plant Biology, 11(3), 417-424. https://doi.org/10.1111/j.14388677.2008.00154.x

Aikpokpodion, P. O. (2012). Assessment of genetic diversity in horticultural and morphological traits among papaya (Carica papaya) accessions in Nigeria. Fruits, 67(3), 173-187. https://doi.org/10.1051/fruits/2012011

Antunes, C. F., Filer, D. \& Renner, S. S. (2014). Cladistics Taxonomy in the electronic age and an e-monograph of the papaya family (Caricaceae ) as an example. Cladistics, 1-9. 
Antunes, C. F. \& Renner, S. S. (2012). A dated phylogeny of the papaya family (caricaceae) reveals the crop's closest relatives and the family's biogeographic history. Molecular Phylogenetics and Evolution, 65, 46-53.

Arias, D., Peñaloza-Ramírez, J., Dorado, O., Cuevas-Reyes, P., Leyva, E., Albarrán-Lara, A. L. \& Rangel-Altamirano, G. (2010). Phylogeographic patterns and possible incipient domestication of Jacaratia mexicana A. DC. (Caricaceae) in Mexico. Genetic Resources and Crop Evolution, 57(8), 1227-1238. https://doi.org/10.1007/s10722010-9569-1

Badillo, V. M. (2000). Carica L. vs. Vasconcella St.-Hil. (Caricaceae) con la rehabilitación de este último. Ernstia, 10, 74-79.

Bullock, S. H. (1992). Seasonal Differences in Nonstructural Carbohydrates in Two Dioecious Monsoon-Climate Trees. Biotropica, 24(2a), 140-145. https://doi.org/10.2307/2388667

Chávez-Pesqueira, M., \& Suárez-Montes, P. Castillo, G. \& Núñez-Farfán, J. (2014). Habitat fragmentation threatens wild populations of Carica papaya (Caricaceae) in a lowland rainforest. American Journal of Botany, 101(7), 1092-1101. https://doi.org/10.3732/ajb.1400051

Cortez-Madrigal, H. \& García-Ruiz, I. (2007). Insectos fitófagos asociados a parientes silvestres de cultivos y plantas raras y endémicas de "Chorros del Varal ", Michoacán, México. Entomología Mexicana, 6(1), 642-647.

Delgado, B. Z. S., Cruz, L. B., Hernández, C. E., Vargas, A. D., Damian, N. A. \& Palemón, A. F. (2016). Evaluación morfológica de diferentes genotipos silvestres y cultivadas de Carica papaya L. en el estado de Guerrero, México. Tlamati, 7(1), 27-30.

Fuentes, G. \& Santamaría, J. M. (2014). Papaya (Carica papaya L.): Origin, domestication, and production. In R. Ming \& P. H. Moore (Ed.), Genetics and genomics of papaya (1st ed., pp. 3-16). Heidelberg Dordrecht London: Springer New York.

García, R. I. \& Linares, L. A. (2012). Árboles y arbustos de la cuenca del rio Tepalcatepec (Michoacán y Jalisco, México) para uso urbano. (El Colegio de Michoacán A. C. e Instituto Politécnico Nacional, Ed.) (1st ed.). México.

Hunter, D. and Heywood, V. (2011). Crop wild relatives: a manual of insitu conservation. (B. International, Ed.). London U.K.: Earthscan.

INEGI. (2016a). Anuario estadístico y geográfico de Michoacán de Ocampo 2016. (Instituto Nacional de Estadística y Geografía, Ed.) (1st ed.). México.

INEGI. (2016b). Anuario estadístico y geográfico de Quintana Roo 2016. (Instituto Nacional de Estadística y Geografía, Ed.) (1st ed.). México.

INEGI. (2016c). Anuario estadístico y geográfico de Tabasco 2016. (Instituto Nacional de Estadística y Geografía, Ed.) (1st ed.). México.

INEGI. (2016d). Anuario estadístico y geográfico del estado de Nayarit 2016. (Instituto Nacional de Estadística y Geografía, Ed.) (1st ed.). México.

Kubitzki, K. (2003). Caricaceae. In K. Kubitzki and C. Bayer (Ed.), Flowering plants. Dicotyledons. The families and genera of vascular plants (1st ed., pp. 57-61). Berlin Heidelberg.

Manshardt, R. (2014). History and future of the solo papaya. In R. Ming \& P. H. Moore (Ed.), Genetics and genomics of papaya (1st ed., pp. 95-113). Heidelberg Dordrecht London.: Springer New York.

Meletti, L. M. M. \& Bruckner, C. H. (2001). Melhoramento genético do maracujazeiro. In M. P. Bruckner, C. H. Coutinho (Ed.), Tecnologia de produção, pós-colheita, agroindústria, mercado (1st ed., pp. 345-385). Maracujá: Porto Alegre: Cinco Continentes.

Niklas, K. J. \& Marler, T. E. (2007). Carica papaya (Caricaceae ): a case study into the effects of growth and reproduction. American Journal of Botany, 94(6), 999-1002.

Paz, L. \& Vázquez-Yanes, C. (1998). Comparative seed ecophysiology of wild and cultivated Carica papaya trees from a tropical rain forest region in Mexico. Tree Physiology, 18(4), 277-280. https://doi.org/10.1093/treephys/18.4.277 


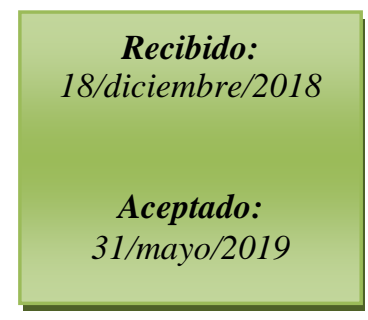

Rodriguez, C. J., Díaz, H. Y., Pérez, G. A., Cruz, Z. N. \& Rodriguez, H. P. (2014). Evaluación de la calidad y el rendimiento en papaya silvestre (Carica papaya L.) de Cuba. Cultivos Tropicales, 35(3), 36-44.

SAS Institute Inc. (2002). The SAS System for Windows 9.0. Cary, N.C. USA.

SNICS-SAGARPA. (2013). Resúmenes ejecutivos, ejercicio fiscal 2010 (p. 223).

SNICS-SAGARPA. (2014). Regla para la calificación de semillas de papaya (Carica papaya L.) (p. 23).

Soriano-Melgar, L. L. A., Alcaraz-Meléndez, L. \& Real-Cosío, S. (2016). Colecta y conservación in vitro y ex situ de recursos fitogenéticos de Carica papaya L. Agroproductividad, 9(4), 28-32.

Teran, S. \& Rasmussen, C. H. (1995). Genetic diversity and agricultural strategy in 16th century and present-day Yucatecan milpa agriculture. Biodiversity and Conservation, 4(4), 363-381. https://doi.org/10.1007/BF00058422 